\title{
Dinámica del daño foliar en plántulas de Drimys granadensis (Winteraceae) y Clusia multiflora (Clusiaceae) en el bosque altoandino de la Cordillera Oriental colombiana
}

\author{
Carolina Ramos ${ }^{1} \&$ Mary R. García ${ }^{2}$ \\ 1. Subdirección Científica, Jardin Botánico Jose Celestino Mutis, Av. Calle 63 No. 68-95, Bogotá, Colombia; charo. \\ ramon@gmail.com \\ 2. Departamento de Biología, Facultad de Ciencias, Universidad Nacional de Colombia; correspondencia: Transv. 34 Bis \\ No 29a-28. Bogotá Colombia; mrgarciaco@unal.edu.co
}

Recibido 23-V-2007. Corregido 24-XII-2007. Aceptado 31-VII-2008.

\begin{abstract}
Leaf damage dynamics of Drimys granadensis (Winteraceae) and Clusia multiflora (Clusiaceae) seedlings in the High-Andean Forest. Because of their slow growth, shade-tolerant species remain exposed to foliar damage long before reproductive age. Foliar damage can be controlled through synchronous leaf production and specialized phytochemical strategies. In this study, the dynamics of foliar damage in different cohorts of seedlings were evaluated to determine if the High-Andean species, Clusia multiflora (Clusiaceae) and Drimys granadensis (Winteraceae) appeal to the synchronous leaf production for controlling the herbivory and pathogenical damage, and to establish the possible relations between the healthy state, growth and mortality of seedlings. Since a recently-emerged seedling could not supply the physiological costs of specialized strategies, we expected the highest synchronous leaf production in the youngest individuals, and differences between cohorts in foliar damage. Four variables were measured to evaluate the health state along time, in three cohorts of seedlings: Proportion of predated leaves $\left(P_{p}\right)$, proportion of healthy leaves $\left(P_{s}\right)$, proportion of leaves with symptoms of disease or punctual damage $\left(P_{e}\right)$ and growing (\% increment of height). Both species showed significant differences between periods in the proportion of healthy leaves, but there was not an effect of time-cohort interaction, therefore synchronic production of leaves was not a strategy more used for any particular cohort. Foliar damage oscillated along time, fact that can be explained by the pulses of leaf production. However, this strategy had little efficiency to control the pathogen attack. In general, the unified behavior of all variables was affected by cohort, time, species an all the different interactions. Association between growing and foliar damage were conditioned by climate. The highest mortality ocurred during the dry season, and one fourth of the D. granadensis deaths were caused by the joint action of herbivory and drought. Results suggest that in spite of the synchronous leaf production in all cohorts, this strategy quickly loses the efficiency for controlling foliar damage, because the means of $P_{s}$ were below of 0.25 at the end of the monitoring period. The differences between species in the dynamics of health state should explain partially their coexistence in the High-Andean forest. Rev. Biol. Trop. 56 (3): 1087-1100. Epub 2008 September 30.
\end{abstract}

Key words: Synchronous leaf production, growth, herbivory, disease, drought, mortality, cohort.

Al tener reducidas tasas de crecimiento, las especies sombra-tolerantes generalmente están expuestas durante mas tiempo al efecto del vecino más cercano, a la limitación de recursos y al daño físico (Feener 1985, Maaron 1997). La acción conjunta o por separado de estos tres factores puede reducir ampliamente la supervivencia durante los primeros meses de desarrollo, o en el menor de los casos, retrasar aún más el ritmo de crecimiento (Silvertown 1987, Swaine 1989, Leigh 1990). Algunos ejemplos documentados del efecto del vecino más cercano abarcan la exposición de plántulas a predadores y microorganismos provenientes del árbol paterno (Janzen 1970, Augspurger 1984, Connell et al. 1984), o de plántulas 
conespecíficas (Gilbert et al. 1994, Hammond y Brown 1998, Meiners y Handel 2000, Sullivan 2003). Las plántulas también sufren altas tasas de mortalidad durante las temporadas de sequía (Fenner 1985, Davis et al. 1999), en las cuales además de verse sometidas a una escasez de nutrientes, son obligadas a reducir la transpiración diurna a costa de la fijación de carbono (Salisbury y Ross 1992, Lüttge 1997, Cavender y Bazzaz 2000).

Existen dificultades para distinguir el daño ocasionado por herbívoros del ocasionado por patógenos. De hecho los dos tipos de daño están asociados, ya que insectos como áfidos y lepidópteros facilitan el transporte y desarrollo de bacterias y hongos a través de la excreta sobre las hojas (Nava y Del Amo 1985, Dirzo 1987, García y Dirzo 2001, Stadler et al. 2001). Gracias a los altos contenidos de agua y nitrógeno de las hojas sin lignificar, las plántulas son preferidas por los fitófagos, aunque su efecto no necesariamente es letal (Coley 1990, Blundell y Peart 2000). En general, los índices de herbivoría en los bosques tropicales son bajos, y su impacto en la demografía sólo se acentúa a través de la interacción con otros factores, como el estrés hídrico y lumínico de la planta, o la competencia inter e intra-específica (Dirzo y Chapin 1987). La supervivencia de una plántula sólo estaría en peligro cuando se consume sus puntos de crecimiento (Dirzo y Harper 1980) o cuando el daño compromete un alto porcentaje del tejido foliar (Blundell y Peart 2001).

Sin duda, la cantidad de biomasa fotosintética es decisiva en el desarrollo exitoso de una plántula y su capacidad de soportar la sequía, y es entones cuando el daño foliar puede hacer la diferencia entre la supervivencia o la muerte de la misma. Mientras las hojas dañadas tienen una mayor probabilidad de absición total (Dirzo 1987, Blundell y Peart 2000, 2001), la producción de nuevas hojas puede ser una reacción de las plántulas frente al daño foliar, aumentando las probabilidades de crecimiento y supervivencia. Una producción sincrónica de nuevas hojas permitiría saciar prontamente a los predadores (Aide 1991, 1993, Kursar y
Coley 2003), asegurando que una proporción de los individuos pase a estados más avanzados del desarrollo. Otras estrategias especializadas contra los herbívoros y patógenos, como la producción de metabolitos secundarios, proteínas de defensa y sustancias volátiles, también han sido ampliamente desarrolladas por las especies sombra-tolerantes (Núñez y Dirzo 1985, Leigh 1990, Baldwin y Preston 1999).

No existe evidencia suficiente acerca de la prontitud con la cual una plántula desarrolla estas estrategias desde el momento de la germinación. Inicialmente las plántulas de especies sombra-tolerantes deben usar las reservas provenientes de la semilla, y producir una biomasa foliar que le permita hacer fotosíntesis bajo deficientes condiciones de luz (Fenner 1983, 1985). El empleo de estrategias especializadas de defensa acarrea además un alto costo fisiológico que requeriría la disponibilidad de agua y nutrientes en el suelo (Leigh 1990, Baldwin y Preston 1999), y en muchos casos, la primera temporada de sequía aparece antes de que la raíz crezca lo suficiente (Garwood 1990, Cavender y Bazzaz 2000). La producción sincrónica de hojas sería entonces la única estrategia que le permitiría a la plántula soportar el daño foliar hasta que ésta esté completamente establecida; pero en el caso particular de los ambientes de montaña, donde además de las condiciones de sombra estas especies deben soportar bajas temperaturas, la plántula podría incluso tardar varios años en establecerse completamente (Halter et al. 1997, Lajzerowicz et al. 2004, Körner y Hoch 2006).

En este estudio se evaluó la dinámica del daño foliar en diferentes cohortes de plántulas de dos especies sombra-tolerantes del bosque altoandino: Clusia multiflora (Clusiaciae) y Drimys granadensis (Winteraceae), con el fin de determinar si la producción sincrónica de hojas es una estrategia de las plántulas frente al daño foliar, y establecer cómo se relaciona el estado de salud con la mortalidad y el crecimiento. En particular nosotros planteamos que existen diferencias entre cohortes en el grado de daño foliar y que la sincronía en la producción de hojas sería más frecuente en los 
individuos más jóvenes, en virtud de que ésta sería la estrategia más económica disponible a partir de la germinación. Las diferencias entre especies, en la dinámica general del estado de salud, sugerirían el uso de estrategias distintas para la supervivencia y ayudaría a explicar su coexistencia en el bosque alto andino.

\section{MATERIALES Y MÉTODOS}

Área de estudio: La zona de estudio incluyó dos bosques localizados en la cordillera Oriental colombiana: Neusa-Laureles $\left(5^{\circ} 08^{\prime} 54^{\prime \prime} \mathrm{N}, 73^{\circ} 57^{\prime} 44^{\prime} \mathrm{O}\right)$ y Monserrate-El Granizo ( $\left.4^{\circ} 37^{\prime} 30^{\prime \prime} \mathrm{N}, 74^{\circ} 02^{\prime} 34^{\prime \prime} \mathrm{O}\right)$, con precipitaciones anuales entre 850 y $1100 \mathrm{~mm}$. Ambos sitios están a una altitud entre 3000 y $3100 \mathrm{msnm}$, presentan una temperatura promedio entre 7 y $10{ }^{\circ} \mathrm{C}$, y están conformados por vegetación de bosque Alto-andino secundario medianamente intervenido, dominado por Clusia multiflora, Drimys granadensis y Weinmannia tomentosa. Estos bosques limitan casi en su totalidad con terrenos de ganadería y cultivos de papa. El régimen climático es similar: Un patrón bimodal de precipitación, con picos en abril-mayo y octubre-noviembre, y temporadas secas en los períodos de diciembre-enero y septiembre (Vargas y Zuluaga 1981, Mora y Sturm 1995, Ballesteros y Rodríguez 1997).

Toma de mediciones para cuantificar el crecimiento y el grado de daño foliar: En septiembre de 2001 se realizó un transecto de $200 \mathrm{~m}$ en cada bosque, sin orientación definida en relación con la pendiente; se censaron y marcaron las plántulas menores de $60 \mathrm{~cm}$ de altura en un área de $1 \mathrm{~m}$ a ambos lados del mismo. El banco de plántulas de Monserrate resultó dominado por Drimys granadensis, mientras en Neusa la proporciones de D. granadensis y Clusia multiflora fueron semejantes.

Para evaluar el daño foliar y el crecimiento de plántulas se empleó un método no destructivo; la longitud $l$ se estimó midiendo las plántulas desde la base del tallo hasta el ápice terminal de la yema de crecimiento; se registró su número de hojas $H_{t}$, el número de hojas con depredación $H_{p}$ y el número de hojas totalmente sanas $H_{s}$.

Las lesiones pequeñas pueden ser ocasionadas por fitófagos o por microorganismos patógenos; por lo tanto, se consideró predada una hoja cuando estaba incompleta en más de un $10 \%$ y no presentaba necrosamiento. Por otro lado, el daño por microorganismos se definió como número de hojas enfermas $H_{e}$, corresponde a las hojas manchas de necrosamiento o con lesiones menores al $10 \%$ del área foliar, y es igual a la diferencia $H_{t}-H_{s}-H_{p}$. En total se realizaron siete muestreos a lo largo de ocho meses de seguimiento.

Análisis estadístico: Con base en las mediciones tomadas, se definieron cuatro variables que estimaban el estado de salud de la plántula, y con las cuales se realizó el análisis estadístico:

Proporción de hojas predadas $P_{p}$ :

$P_{p}=H_{p} / H_{t}$

Proporción de Hojas sanas $P_{s}$ :

$P_{s}=H_{s} / H_{t}$

Proporción de Hojas enfermas $P_{e}$ :

$P_{e}=H_{e} / H_{t}$

Crecimiento, expresado como el incremento en porcentaje de la altura:

$\%$ crecimiento $=\sum_{0}^{i} \frac{\Delta l \times 100}{l_{i-1}}$

donde $\Delta l=l_{i}-l_{i-1}$ es el incremento en altura del $i$-ésimo registro.

Las variables $P_{p}, P_{s}$ y $P_{e}$ presentaron valores de 0 a 1 que cumplieron la normalidad de Kolmogorov-Smirnov, (Sokal y Rohlf 1997) y la homogeneidad de varianzas de Bartlett (Zar 1999). En algunos casos se requirió la transformación de datos tipo $\log _{10}(x+1)$. Para evaluar los efectos de la herbivoría y la enfermedad entre cohortes distintas, las poblaciones de plántulas fueron diferenciadas en tres ámbitos de edad, teniendo en cuenta que cada grupo tuviese un tamaño muestral representativo. 
Para plántulas de C. multiflora en el bosque de Neusa: menores de $15 \mathrm{~cm}$, de 15 a $30 \mathrm{~cm}$ y de 30 a $60 \mathrm{~cm}$. Para plántulas de D. granadensis en el bosque de Monserrate: menores de $8 \mathrm{~cm}$, entre 8 y $16 \mathrm{~cm}$ y de 16 a $32 \mathrm{~cm}$. Las plántulas de esta especie en Neusa sufrieron una fuerte reducción poblacional durante el tiempo de muestreo, y fueron eliminadas del estudio debido a una baja representatividad estadística.

Las diferencias entre cohortes para las diferentes variables fueron evaluadas por registro mediante una prueba t para diferencia de medias, y a lo largo del tiempo con un ANOVA de medidas repetidas. La diferencia entre especies en el comportamiento de todas las variables, se analizó mediante un MANOVA, y se calcularon coeficientes de correlación de Spearman en las diferentes temporadas climáticas para determinar si existe una relación entre el crecimiento y el daño foliar. Finalmente se determinó el modelo de regresión para la supervivencia de plántulas de cada especie (Statistica 6.0 - Stat Soft. Inc. 1997, SAS L.E. 1.3.0.161 - SAS Institute Inc. 2000).

Determinación de causas de mortalidad: El número de plántulas muertas fue registrado a lo largo del tiempo de muestreo, y en cada caso se asignó una causa de muerte, de acuerdo a los siguientes criterios:

\section{Causa de muerte:}

Herbivoría: Individuos que mueren durante temporada húmeda y que cumplían con la condición $P_{e}<0.3$ y $P_{p} \geq 0.5$.

Sequía: Individuos que no sobrevivieron a la época seca, aunque sus registros previos cumplían la condición $P_{s}>0.6$.

Depredación y Sequía: Individuos que mueren durante la época seca, pero con registros previos que cumplían la condición $P_{e}<0.3$ y $P_{p} \geq 0.5$.

Enfermedad: Individuos que mueren durante la época húmeda, con señales de necrosamiento o daño puntual no definido, cumpliendo la condición $P_{e} \geq 0.6$ y $P_{p}<0.3$.

Enfermedad y depredación: Individuos que mueren durante la época húmeda y que cumplían con la condición $P_{p} \geq 0.4$ y $P_{e}$ $\geq 0.4$.

Sepultamiento: Individuos que desaparecen tras ser sepultados por arrastre o caída de material vegetal, como bromelias, ramas y hojarasca.

\section{RESULTADOS}

Dinámica del daño foliar y crecimiento: La dinámica del daño foliar en todas las cohortes presentó un patrón sincrónico con valores oscilantes en el tiempo en ambas especies, exceptuando el crecimiento. En ambas especies la dinámica del daño foliar y la producción de hojas tuvo una tendencia sincrónica de las cohortes, con rasgos oscilantes a través del tiempo (Figs. 1 y 2). En plántulas de C. multiflora, las media general de $P_{p}$ tuvo sus máximos en octubre $(0.40 \pm 0.04)$ y mayo $(0.39 \pm 0.04)$, y mostró descensos en noviembre $(0.31 \pm 0.04)$ y marzo $(0.25 \pm 0.03)$. En noviembre se dio además un pico sincrónico de crecimiento (incremento promedio de $4.53 \%$ a $6.24 \%$ ), y las medias de $P_{e}$ tuvieron sus máximos en marzo $(0.64 \pm 0.04)$. Al final del seguimiento prácticamente la totalidad de las plántulas de ésta especie presentaban un promedio de $P_{s}$ cercano a cero $(0.04 \pm 0.02)$. En plántulas de $D$. granadensis, las más altas medias de depredación se dieron en marzo $\left(P_{p}=0.48 \pm 0.05\right)$, y los descensos más drásticos de $P_{s}$ tuvieron lugar entre marzo y abril $(0.09 \pm 0.02)$. Los promedios de la variable $P_{e}$ también mostraron picos sincrónicos, durante noviembre $(0.40 \pm 0.05)$ y junio $(0.48 \pm 0.05)$.

Durante gran parte del seguimiento, las plántulas más grandes de C. multiflora (30$60 \mathrm{~cm})$ mostraron los más altos índices de depredación $\left(P_{p}\right.$ entre 0.374 y 0.562$)$, los menores porcentajes de crecimiento $(1,82 \%$ al 3,16\%), y las más bajas proporciones de hojas enfermas $\left(P_{e}\right.$ entre 0.36 y 0.527$)$ (Fig. 1). Sin embargo, las diferencias entre cohortes no fueron significativamente consistentes a través del tiempo (Proporción de hojas predadas: $\mathrm{F}_{2,27}=0.779, \mathrm{p}=0.469$; proporción de hojas sanas: $\mathrm{F}_{2,27}=0.888, \mathrm{p}=0.420$; proporción 


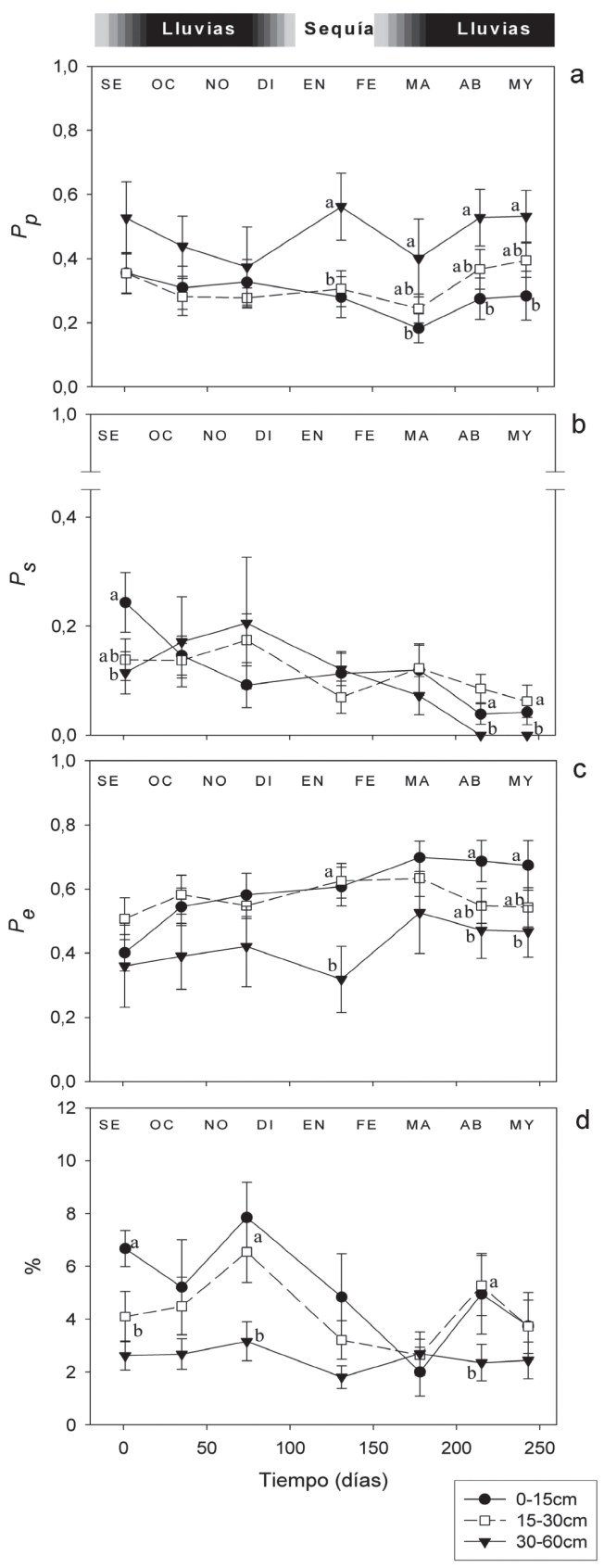

Fig. 1. Dinámica del estado de salud en plántulas de Clusia multiflora (media $\pm 1 \mathrm{ES})$. a) Depredación, b) hojas sanas, c) hojas enfermas, d) crecimiento. Letras distintas en un mismo registro indican diferencias entre cohortes.

Fig. 1. Dynamic of seedlings health of Clusia multiflora (mean $\pm 1 \mathrm{SE}$ ). a) Predation, b) healthy leaves, c) leaves with symptoms of disease, d) growth. Different letters in the same date represent differences among cohorts.

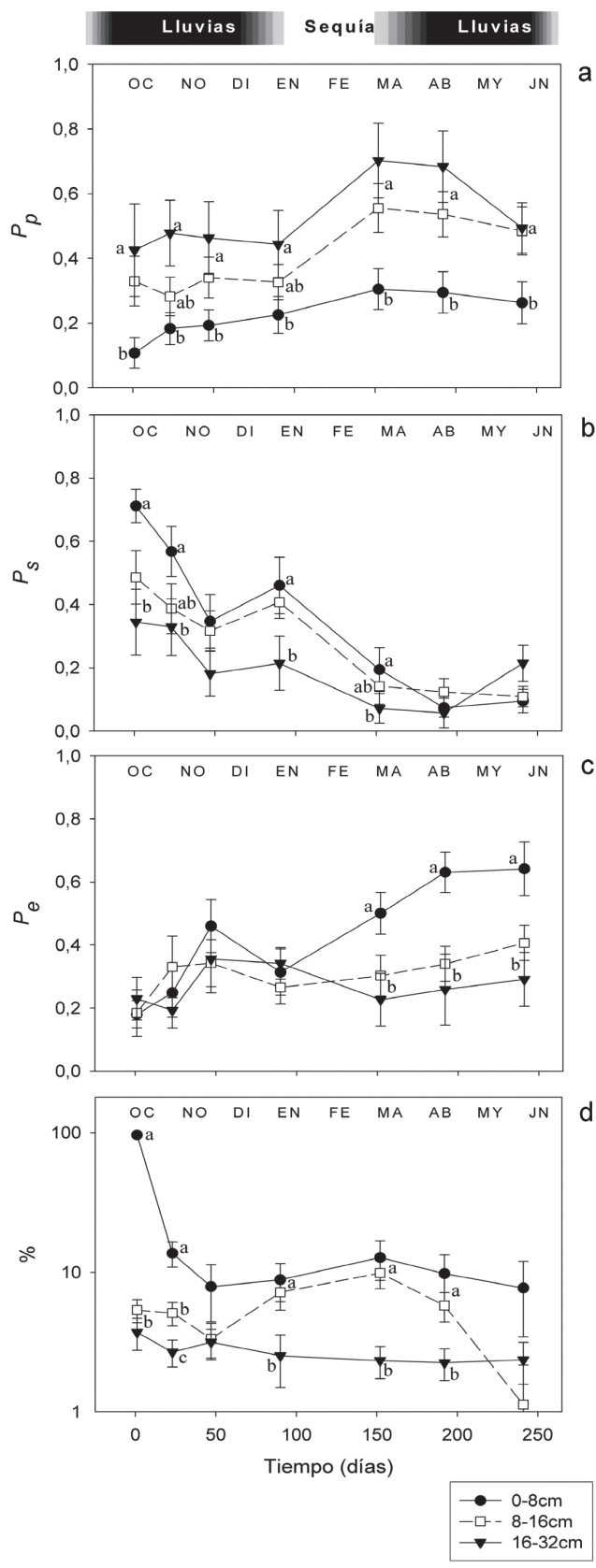

Fig. 2. Dinámica del estado de salud en plántulas de Drimys granadensis (media \pm 1 ES). a) Depredación, b) hojas sanas, c) hojas enfermas, d) crecimiento. Letras distintas en un mismo registro indican diferencias entre cohortes.

Fig. 2. Dynamic of seedling health of Drimys granadensis (mean $\pm 1 \mathrm{SE}$ ). a) Predation, b) healthy leaves, c) leaves with symptoms of disease, d) growth. Different letters in the same date represent differences between cohorts. 
de hojas enfermas: $\mathrm{F}_{2,27}=0.972, \mathrm{p}=0.388$; crecimiento: $\mathrm{F}_{2,27}=2.776, \mathrm{p}=0.075$ ).

D. granadensis mostró claras diferencias entre cohortes, en la proporción de hojas predadas $\left(\mathrm{F}_{2,34}=5.637, \mathrm{p}=0.008\right)$ y sanas $\left(\mathrm{F}_{2,34}=3.308\right.$, $\mathrm{p}=0.049)$. La cohorte más antigua $(16-32 \mathrm{~cm})$ tuvo promedios de depredación mayores $(0.425$ en septiembre a 0.702 en mayo) a los de la cohorte más joven $(0-8 \mathrm{~cm}) P_{p}$ entre 0.108 y 0.305), y mostró además los menores valores de proporción de hojas sanas. En esta especie, la variable $P_{s}$ tuvo una tendencia general decreciente (de 0.555 a 0.088 ). La enfermedad en hojas fue mayor en las plántulas más pequeñas durante la época húmeda y en la cohorte más antigua se dieron los menores ritmos de crecimiento (Fig. 2), pero en ninguna de estas variables el efecto de la cohorte fue significativo a través del tiempo (Proporción de hojas enfermas: $\mathrm{F}_{2,34}=0.992, \mathrm{p}=0.381 ; \%$ Crecimiento: $\mathrm{F}_{2,34}=2.933, \mathrm{p}=0.067$ ).

\section{Relación entre el crecimiento y el daño}

foliar: La relación entre el crecimiento y los indicadores de salud $\left(P_{p}, P_{e}\right.$ y $\left.P_{s}\right)$ varió entre cohortes. En el caso de C. multiflora, El crecimiento de las plántulas más pequeñas no tuvo ninguna correlación con el daño foliar; las plántulas de tamaño intermedio mostraron una relación negativa entre el crecimiento y la proporción de hojas sanas $\left(\mathrm{r}_{\mathrm{S}}=-0.197 \mathrm{p}=0.031\right.$; $\mathrm{N}=119$ ); en los individuos más grandes el crecimiento se relacionó positivamente con la proporción de hojas enfermas $\left(\mathrm{r}_{\mathrm{S}}=0.326 \mathrm{p}=0.012\right.$; $\mathrm{N}=59)$ y negativamente con la herbivoría $\left(\mathrm{r}_{\mathrm{S}}=\right.$ $0.276 \mathrm{p}=0.034 ; \mathrm{N}=59$ ).

En $D$ granadensis, sólo la cohorte de $0-8 \mathrm{~cm}$ presentó una asociación entre el crecimiento y el daño foliar, y ésta varió entre épocas climáticas: Durante la época húmeda los individuos con menor crecimiento tuvieron mayores valores de $P_{e} \quad\left(\mathrm{r}_{\mathrm{S}}=-0.383 \mathrm{p}<0.001\right.$; $\mathrm{N}=73)$ y menores valores de $P_{s}\left(\mathrm{r}_{\mathrm{S}}=0.398\right.$ $\mathrm{p}<0.001 ; \mathrm{N}=73$ ). Durante la época seca el crecimiento de esta misma cohorte estuvo positivamente relacionado con la depredación $\left(\mathrm{r}_{\mathrm{S}}=0.425 \mathrm{p}=0.017 ; \mathrm{N}=31\right)$ y negativamente relacionado con la proporción de hojas sanas $\left(\mathrm{r}_{\mathrm{S}}=-0.476 \mathrm{p}=0.007 ; \mathrm{N}=31\right)$.
Efectos generales sobre el estado de salud de las plántulas: El MANOVA mostró efectos altamente significativos de la especie, la cohorte, el tiempo y de todas interacciones sobre el comportamiento general de la salud de las plántulas (Respuesta total; Cuadro 1). Sin embargo, al descomponer estos efectos en las diferentes variables, sólo el crecimiento mostró diferencias significativas en función de todos los factores e interacciones (Cuadro 1, Fig. 3). Las plántulas más pequeñas de $D$. Granadensis crecieron a ritmos del doble del promedio general para la especie (crecimiento 0-8cm: $22,53 \pm 3,36 \%$; media de crecimiento para la especie: $12,29 \pm 1,59 \%$ ), y cinco veces el promedio general para $C$. multiflora (media de crecimiento: 4,16 $\pm 0,19 \%$ ). Entre especies no hubo diferencias en la proporción de hojas predadas, pero sí en la proporción de hojas sanas $\left(P_{s}=0.11 \pm 0.01\right.$ en $C$. multiflora, $P_{s}=0.29 \pm 0.02$ en $D$. granadensis) y de hojas enfermas $\left(P_{e}=0.55 \pm 0.02\right.$ en $C$. multiflora, $P_{e}=0.35 \pm 0.02$ en $D$. granadensis $)$. El efecto del tiempo y la cohorte fue significativo, pero para la proporción de hojas predadas y sanas, estos efectos además dependieron de la especie.

Causas de mortandad: La dinámica de la mortandad de plántulas fue distinta entre especies (Fig. 4). La pérdida de individuos de C. multiflora se dio a ritmos casi constantes, que se ajustaron a un modelo de regresión lineal negativa $(\mathrm{p}<0.01)$, con sólo un total de 6 plántulas muertas $(12,5 \%)$, de los cuales 4 pertenecieron a la cohorte intermedia. Por otra parte, la mortandad de $D$. granadensis se ajustó a un modelo exponencial negativo $(\mathrm{p}<0.01)$, con un conteo total de 20 individuos muertos (30,8\%), de los cuales 15 pertenecían a la cohorte más joven. Las principales causas de mortandad de $D$. granadensis fueron la sequía y la acción conjunta de la sequía y la depredación, mientras un $67 \%$ de la mortandad en plántulas de C. multiflora estuvo relacionada con el ataque microbiano y el daño puntual (Cuadro 2). En total, la mortandad asociada a herbivoría, acumulada para las dos especies 
CUADRO 1

Efecto de la especie, la edad y el tiempo sobre la depredación, las hojas sanas, las hojas enfermas, el crecimiento y la respuesta general de la salud de las plántulas

TABLE 1

Effects of species, age and time on the predation, healthy leaves, leaves with symptoms of damage, growth and general response of seedlings

\begin{tabular}{|c|c|c|c|c|c|c|c|c|c|c|}
\hline \multirow{2}{*}{ Efecto } & \multicolumn{2}{|c|}{$P_{p}$} & \multicolumn{2}{|c|}{$P_{s}$} & \multicolumn{2}{|c|}{$P_{e}$} & \multicolumn{2}{|c|}{ Crecimiento } & \multicolumn{2}{|c|}{ Total (MANOVA) } \\
\hline & $\mathrm{F}$ & $\mathrm{p}$ & $\mathrm{F}$ & $\mathrm{p}$ & $\mathrm{F}$ & $\mathrm{p}$ & $\mathrm{F}$ & $\mathrm{p}$ & $\mathrm{F}$ & $\mathrm{p}$ \\
\hline Especie & 1.19 & 0.28 & 90.85 & $<0.01$ & 70.06 & $<0.01$ & 94.81 & $<0.01$ & 77.36 & $<0.01$ \\
\hline Cohorte & 36.23 & $<0.01$ & 6.50 & $<0.01$ & 15.57 & $<0.01$ & 123.11 & $<0.01$ & 50.28 & $<0.01$ \\
\hline Tiempo & 2.21 & 0.04 & 20.31 & $<0.01$ & 5.27 & $<0.01$ & 47.35 & $<0.01$ & 21.15 & $<0.01$ \\
\hline Especie* Cohorte & 4.91 & 0.01 & 4.37 & 0.01 & 2.11 & 0.12 & 86.91 & $<0.01$ & 31.02 & $<0.01$ \\
\hline Especie* Tiempo & 3.96 & $<0.01$ & 7.04 & $<0.01$ & 0.97 & 0.45 & 44.58 & $<0.01$ & 16.60 & $<0.01$ \\
\hline Cohorte*Tiempo & 0.52 & 0.90 & 1.71 & 0.061 & 1.69 & 0.07 & 52.78 & $<0.01$ & 14.24 & $<0.01$ \\
\hline Especie*Cohorte*Tiempo & 0.28 & 0.93 & 1.19 & 0.284 & 0.71 & 0.74 & 48.46 & $<0.01$ & 12.97 & $<0.01$ \\
\hline
\end{tabular}
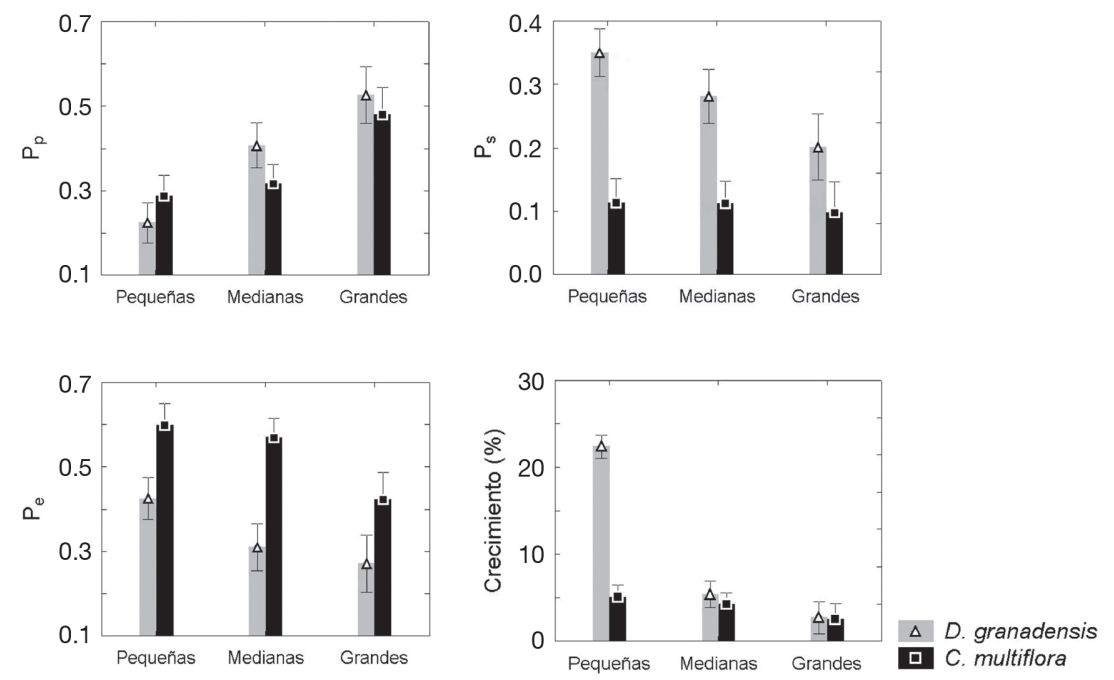

Fig. 3. Diferencias en la salud de las plántulas, entre especies y entre cohortes de la misma especie (medias e intervalos del $95 \%$ de confianza).

Fig. 3. Differences in seedling health, between species and between cohorts from the same species (means and $95 \%$ confidence intervals). 


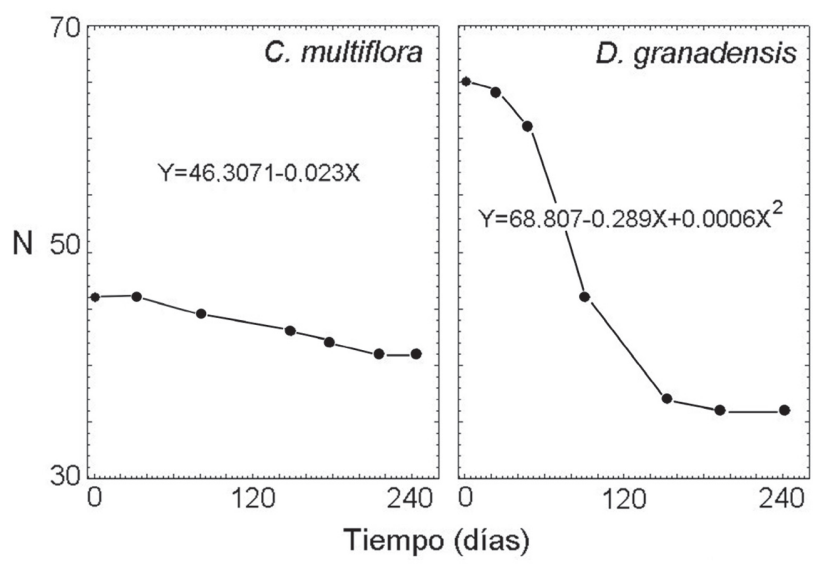

Fig. 4. Supervivencia de plántulas a lo largo del tiempo de seguimiento. La regresión se ajustó a un modelo lineal para $C$. multiflora y un modelo no lineal para D. granadensis.

Fig. 4. Seedlings survival along time. Regression was fitted to a lineal model in C. multiflora and a non-lineal model in $D$. granadensis.

(17,57\%), superó el doble a aquella asociada a la enfermedad $(8,32 \%)$.

\section{DISCUSIÓN}

Los resultados permitieron caracterizar la dinámica del daño foliar y la salud de plántulas de $C$. multiflora y $D$. granadensis en el bosque altoandino colombiano. En las plántulas de $C$. multiflora, la depredación fue similar al inicio $\mathrm{y}$ al final del muestreo, lo cual sugiere que la herbivoría tiende a mantenerse bajo control sin importar la cohorte. Un patrón semejante se dio en la cohorte más antigua de D. granadensis, mientras que un aumento de la depredación en las plántulas jóvenes e intermedias (del 150\% y $50 \%$ respectivamente; Figs. 1 y 2 ) indica que las estrategias de control en esas cohortes no son lo suficientemente eficientes. Por otra parte, se observó una reducción neta de la proporción de hojas sanas en ambas especies durante el tiempo de seguimiento (reducción del $77,19 \%$ en $D$. granadensis y de $77,44 \%$ en $C$. multiflora), la cual estaría más relacionada con un aumento en la enfermedad de las hojas y daño puntual, que con la herbivoría (Figs. 1 y 2).

Los patrones oscilantes en la dinámica del estado de salud de las plántulas, indi- can que existen temporadas de producción de hojas, y que éstas son sincrónicas entre las cohortes estudiadas, estrategia que de acuerdo con Fenner (1985) y Dirzo y Chapin (1987), resulta efectiva para lograr la saciedad de los herbívoros. En las plántulas más antiguas los patrones de oscilación del daño foliar $\left(P_{p}\right.$ y $\left.P_{e}\right)$ fueron más acentuados que la producción de hojas $\left(P_{s}\right)$, mientras las plántulas más jóvenes mostraron la tendencia opuesta. En el caso de C. multiflora, este comportamiento llevó a una proporción de hojas sanas igual a cero en los últimos registros para la cohorte mayor, lo cual indica que la producción sincrónica de hojas funciona como estrategia de control sólo durante las primeras etapas de vida. (Cuadro 2).

En $D$. granadensis la cohorte mayor mostró un aumento en los valores de $P_{s}$ hacia el final del tiempo de seguimiento, lo cual sugiere que tiene la capacidad de mantener una producción más eficiente de hojas nuevas (Fig. 2). En el bosque del Neusa se observó que un tercio de las plántulas más grandes de esta especie $(>20 \mathrm{~cm})$ desarrollaban rebrotes de rápido crecimiento, particularmente cuando la depredación era intensa e involucraba la yema terminal. En general, D. granadensis es reconocida por su capacidad de presentar regeneración vegetativa 
CUADRO 2

Mortandad de plántulas por cohorte y especie

TABLE 2

Seedling mortality per species and cohort

\begin{tabular}{|c|c|c|c|c|c|c|c|c|}
\hline \multirow[b]{3}{*}{ Causa de mortandad } & \multicolumn{8}{|c|}{ Mortandad (\%) } \\
\hline & \multicolumn{4}{|c|}{ C. multiflora } & \multicolumn{4}{|c|}{ D. granadensis } \\
\hline & $\begin{array}{l}0-15 \mathrm{~cm} \\
(\mathrm{~N}=15)\end{array}$ & $\begin{array}{c}15-30 \mathrm{~cm} \\
(\mathrm{~N}=18)\end{array}$ & $\begin{array}{c}30-60 \mathrm{~cm} \\
(\mathrm{~N}=10)\end{array}$ & $\begin{array}{l}\text { TOTAL } \\
(\mathrm{N}=48)\end{array}$ & $\begin{array}{c}0-8 \mathrm{~cm} \\
(\mathrm{~N}=24)\end{array}$ & $\begin{array}{l}8-16 \mathrm{~cm} \\
(\mathrm{~N}=28)\end{array}$ & $\begin{array}{c}16-32 \mathrm{~cm} \\
(\mathrm{~N}=13)\end{array}$ & $\begin{array}{l}\text { TOTAL } \\
(\mathrm{N}=65)\end{array}$ \\
\hline Sequía & 0 & 0 & 0 & 0 & 13.84 & 3.08 & 0 & 16.92 \\
\hline Sequía + Depredación & 0 & 2.08 & 0 & 2.08 & 4.62 & 3.08 & 0 & 7.70 \\
\hline Depredación & 0 & 0 & 0 & 0 & 1.54 & 0 & 0 & 1.54 \\
\hline Sepultamiento & 2.08 & 0 & 0 & 2.08 & 3.08 & 1.5 & 0 & 4.58 \\
\hline $\begin{array}{l}\text { Enfermedad + } \\
\text { Depredación }\end{array}$ & 2.08 & 2.08 & 2.08 & 6.25 & 0 & 0 & 0 & 0 \\
\hline Enfermedad & 0 & 4.16 & 0 & 4.16 & 0 & 0 & 0 & 0 \\
\hline TOTAL & 4.16 & 6.25 & 2.08 & 12.50 & 23.10 & 7.69 & 0 & 30.79 \\
\hline
\end{tabular}

en múltiples formas desde tempranos estados del desarrollo (Vargas 1986), estrategia útil para compensar el daño foliar. Aunque hay evidencia de que la especie produce metabolitos secundarios contra áfidos y lepidópteros (Ciccio 1997), los aumentos en daño foliar en las cohortes menor e intermedia sugieren que estas sustancias sólo se producen en estados posteriores de desarrollo.

C. multiflora presenta hojas coriáceas altamente lignificadas que las hace poco palatables para los herbívoros. La proporción de hojas predadas en esta especie llegó a un valor máximo de 0.56 en febrero (cohorte $30-60 \mathrm{~cm}$, Fig. 1) y no hubo un aumento considerable de la depredación a lo largo del tiempo de seguimiento, ni siquiera para la cohorte mayor. Existen además reportes de la presencia de diferentes alcaloides de acción anti-alimentaria e insecticida en las hojas de esta especie (Moreno y Luque 1995) y otros metabolitos secundarios en la subfamilia Clusoidae (García y Morales 1997) que funcionarían como estrategia de defensa frente a los predadores. Otros estudios han demostrado que las hojas de C. multiflora tienen un ritmo muy lento de senescencia, que les permite

permanecer funcionalmente en la planta hasta por un tiempo de dos años (Olivares 1997).

Aunque se podría pensar que la producción de hojas sería favorecida por el crecimiento, o viceversa, realmente esto depende de la especie y la temporada climática. Los datos de correlación indicaron que las plántulas intermedias de C. multiflora con menor proporción de hojas crecieron más, al igual que los individuos más jóvenes de $D$. granadensis durante la época seca. Esto podría ser explicado por las bajas tasas de renovación de hojas en C. multiflora y la necesidad de condiciones de humedad para la fabricación de nuevas hojas en $D$. granadensis. De hecho, durante la temporada húmeda las mismas plántulas de ésta especie mostraron una relación positiva y significativa entre el crecimiento y la variable $P_{s}$.

El aumento del daño foliar en plántulas de C. multiflora durante la época seca coincidió con una importante disminución del crecimiento promedio en todas las cohortes. Los individuos mas pequeños y con mayor crecimiento tendieron a mostrar los mayores índices de depredación, pero en un estado mas avanzado de desarrollo el crecimiento estuvo más relacionado con la enfermedad que con la 
herbivoría; de tal modo que hacia el final del muestreo todas las plántulas antiguas presentaban tasas bajas de crecimiento y la totalidad de sus hojas con algún tipo de lesión. En $D$. Granadensis la depredación también aumentó durante la época seca, aunque no se encontró un patrón general de relación entre el daño foliar y el crecimiento. La cohorte mayor tuvo mayores índices de herbivoría que las plántulas de la cohorte más joven, pero fueron éstas las que tuvieron comprometido su crecimiento por la herbivoría y la enfermedad.

La sequía, sin previo daño foliar, fue la principal causa de mortandad en $D$. granadensis, con un $82 \%$ correspondiente a la cohorte más joven $(0-8 \mathrm{~cm})$. Por otra parte, $C$. multiflo$r a$ mostró un patrón homogéneo de supervivencia, superando exitosamente esta temporada; su morfología suculenta en tallo y hojas es una adaptación que le permite superar el estrés hídrico. Aunque no es considerada CAM facultativa, como varias especies del género Clusia (Holtum, et al., 2004), C. multiflora es capaz además de mantener bajos valores de $\mathrm{pH}$ vacuolar (Olivares 1997), lo cual le permitiría modificaciones temporales de la ruta de fijación de $\mathrm{CO}_{2}$ para reducir la pérdida de agua.

El daño foliar es considerado una de las principales causas de mortandad de plántulas de especies arbóreas (Tozer y Bradstock 1997, Gilbert 2002, Li y Ma 2003). En la mayoría de los casos, la herbivoría causa una mayor mortandad si las plántulas están presentes en altas densidades (Hammond y Brown 1998, Meiners y Handel 2000), escenario típico de las cohortes más jóvenes. En este estudio, la depredación por si sola no fue una causa importante de mortandad (1.54\% en D. granadensis, $0 \%$ en $C$. multiflora), pero una cuarta parte de las muertes de $D$. granadensis fue causada por la interacción entre herbivoría y sequía, es decir que estos individuos no sobrevivieron a la época seca y a su vez mostraban valores importantes de $P_{p}$ (Cuadro 2). Otros estudios coinciden en asociar la sequía con aumentos en las tasas de consumo de hojas jóvenes, y la consiguiente mortandad de plántulas (Coley 1990, Meiners et al. 2000, Tesfaye et al. 2002).
El ambiente seco actuaría en dos formas: estimulando el crecimiento de las poblaciones de insectos, y causando estrés hídrico en las plantas, haciéndolas menos tolerantes a la depredación. Con la reducción de área foliar también se reduce el transporte de agua y nutrientes a través del tallo (Tyree et al. 1998, Cavender y Bazzaz 2000) y aumenta la probabilidad de morir durante la época seca.

La enfermedad, descrita como lesiones puntuales, manchas y necrosis, tuvo un patrón general de aumento en ambas especies (Figs. 1 y 2), lo cual indica que este tipo de daño es pobremente controlado por la producción de hojas nuevas, y confirma que el sotobosque tropical es uno de los ambientes que más favorece el ataque por patógenos (Augspurger 1983). Sin importar la especie, los mayores promedios en la proporción de hojas enfermas se dieron en la cohorte más joven durante la segunda temporada húmeda. Cuatro de seis muertes en $C$. multiflora estuvieron asociadas a enfermedad en las hojas, mientras D. granadensis no mostró mortandad causada por daño microbiano (Cuadro 2). Con todo, la mortandad causada por microorganismos, asociada o no a la depredación sólo representó el 8,3\% de la población de plántulas de C. multiflora, a pesar de los reportes de mortandad superiores al $40 \%$ en comunidades vegetales tropicales debidos a patógenos y fitófagos (García y Dirzo 2001).

Se comprobó que el comportamiento general de las plántulas frente al daño foliar fue significativamente distinto entre especies, entre cohortes y entre fechas (Cuadro 1, Fig. 3). Al estudiar los efectos por separado sobre cada variable respuesta $\left(P_{p}, P_{s}, P_{e}\right.$ y crecimiento) sólo la cohorte y el tiempo tuvieron efectos significativos en todos los casos, lo cual demuestra la importancia de considerar estos factores en estudios de regeneración de especies arbóreas.

La hipótesis de que existe una producción sincrónica de hojas en plántulas de estas especies se demostró mediante el MANOVA, con diferencias entre períodos de muestreo en la proporción de hojas sanas. Este efecto tuvo una significancia mucho mayor al efecto 
que tuvo el tiempo sobre las variables de daño foliar, lo cual puede sugerir que los pulsos en la producción de hojas serían un estimulador de la dinámica cambiante del daño foliar. Sin embargo, el hecho de que no exista un efecto de la interacción cohorte y tiempo sobre la variable $P_{s}$ indica que no hay una edad en particular en la que la plántula presente mayor sincronía que las demás.

En síntesis, C. multiflora y D. granadensis responden en forma distinta frente al daño foliar. Aunque ambas especies recurren inicialmente a la producción sincrónica de hojas, esta estrategia se dejaría de lado cuando las plántulas de C. multiflora aumentan de tamaño, abriendo paso a las adaptaciones foliares y a la producción de sustancias especializadas. Sin embargo, la baja tasa de renovación de sus hojas la hace más susceptible al ataque microbiano. D. granadensis emplearía la temprana producción de renuevos para enfrentar a la herbivoría, lo cual retrasa el ataque de patógenos a las hojas, pero es menos efectivo en el control de los fitófagos. El balance general indica que C. multiflora presenta índices de hojas sanas entre dos y tres veces más bajos, pero la mortandad de $D$. granadensis es 2,5 veces mayor (Fig. 3, Cuadro 2). Estas dos especies terminan creciendo a ritmos similares y usan los mismos ambientes dentro del sotobosque, por lo tanto los herbívoros podrían cumplir un papel muy importante en el bosque altoandino, permitiendo su coexistencia (Connell 1971, Leight 1990, Hulme 1996).

En conclusión, existen diferencias en el daño foliar entre cohortes distintas de plántulas. La herbivoría asociada a la sequía causó un importante porcentaje de mortandad en $D$. granadensis, mientras la pérdida de reclutas de C. multiflora estuvo más relacionada con la enfermedad que con la depredación. Se comprobó que estas especies recurren a la producción sincrónica de hojas como un medio de compensación del daño foliar y el comportamiento general de las plántulas frente al daño foliar fue distinto entre especies, y entre temporadas climáticas, y esta variabilidad favorecería la coexistencia en el bosque altoandino. Sería recomendable tener en cuenta el microclima y la dinámica de las poblaciones de herbívoros en estudios de reclutamiento de especies nativas.

\section{AGRADECIMIENTOS}

El presente estudio se realizó gracias al financiamiento de los programas 803583 y 803637 de la División de Investigación de la Universidad Nacional (DIB). También agradecemos la invaluable colaboración y asesoría de Eduardo Narbona, y la contribución de los investigadores Hernán Cardozo y David Rivera.

\section{RESUMEN}

Además de las estrategias fitoquímicas especializadas, las plantas pueden usar la producción sincrónica de hojas como un método de saciado de patógenos y herbívoros. Con el fin de determinar si las especies del bosque altoandino colombiano Clusia multiflora (Clusiaceae) y Drimys granadensis (Winteraceae) recurren a la producción sincrónica de hojas para controlar los efectos de la herbivoría y el ataque microbiano, y establecer que relación existe entre el estado de salud, el crecimiento y la mortalidad de plántulas, se evaluó la dinámica del daño foliar sobre diferentes cohortes. Dado que una plántula tolerante a la sombra recientemente emergida no puede suplir los costos fisiológicos de una estrategia especializada, se esperaba una alta sincronía en la producción de hojas en las plántulas más jóvenes, y diferencias en el daño foliar entre cohortes. Se midieron cuatro variables que evaluaban el estado de salud a lo largo del tiempo, en tres cohortes de plántulas: Proporción de hojas predadas, proporción de hojas sanas, proporción de hojas enfermas o con daño puntual y crecimiento. Ambas especies mostraron diferencias significativas entre épocas, en la proporción de hojas sanas; pero no hubo un efecto de la interacción tiempo-cohorte, por lo tanto la producción sincrónica de hojas no fue una estrategia más usada por alguna cohorte en particular. El daño foliar osciló a través del tiempo, lo cual puede ser explicado por los pulsos en la producción de hojas. Sin embargo, ésta estrategia tuvo poca eficiencia para controlar el ataque por patógenos. En general, el comportamiento unificado de todas las variables fue afectado por la cohorte, el tiempo, la especie y todas las diferentes interacciones. La relación entre crecimiento y daño foliar fue condicionado por el clima. La mayor mortalidad se dio durante la estación seca, y un cuarto de las muertes en D. granadensis fueron causadas por la acción conjunta de herbivoría y sequía. Los resultados sugieren que a pesar de la producción sincrónica de hojas en todas las cohortes, fue poca la eficiencia para controlar el daño foliar, ya que 
las medias de $P_{s}$ estuvieron por debajo de 0.25 al final del seguimiento. Las diferencias en la dinámica del estado de salud entre especies podrían explicar parcialmente la coexistencia en el bosque altoandino.

Palabras clave: Producción sincrónica de hojas, crecimiento, herbivoría, enfermedad, sequía, mortalidad, cohorte.

\section{REFERENCIAS}

Aide, T.M. 1991. Synchronous leaf production and herbivory in juveniles of Gustavia superba. Oecologia 88:511-514.

Aide, T.M. 1993. Patterns of leaf production and herbivory in a tropical understory community. Ecology 74 455-466.

Augspurger, C.K. 1983. Seed dispersal of the tropical tree, Platypodium elegans, and the escape of its seedlings from fungal pathogens. J. Ecol. 71: 759-771.

Augsurger, C.K. 1984. Seedling survival of tropical tree species: interactions of dispersal distance, light-gaps, and pathogens. Ecology 65: 1705-1712.

Baldwin, I.T. \& C.A. Preston. 1999. The eco-physiological complexity of plant responses to herbivores. Planta 208: 137-145.

Ballesteros, M. \& J. Rodríguez. 1997. Diversidad Biológica y Funcionamiento del Bosque altoandino Circundante al Embalse del Neusa (Cundinamarca). Informe de Investigación. Fondo FEN. Bogotá, Colombia.

Blundell, A. \& D. Peart. 2000. High abscission rates of damaged expanding leaves: field evidence from seedlings of a Bornean rain forest tree. Am. J. Bot. 87: 1693-1698.

Blundell, A. \& D. Peart, 2001. Growth strategies of a shade-tolerant tropical tree: the interactive effects of canopy gaps and simulated hervibory. J. Ecol. 89: 608-615.

Cavender, J. \& F. Bazzaz. 2000. Changes in drought response strategies with ontogeny in Quercus rubra: implications for scaling from seedlings to mature trees. Oecologia 124: 8-18.

Ciccio, J.F. 1997. Aceites esenciales de las hojas y de los frutos verdes de Drimys

granadensis (Winteraceae). Rev. Biol. Trop. 44-45: 29-33.

Connell, J.H. 1971. On the role of natural enemies in preventing competitive exclusion in some marine animals and in rain forest trees. p. 298-312. In: J.P. Den Boer \& G.R.Gradwell (eds). Dynamics of populations. Centre for Agricultural Publication and Documentation, Wageningen, Holanda.

Connell, J.H., J.G. Tracey, \& L.J. Webb. 1984. Compensatory recruitment, growth, and mortality as factors maintaining rain forest tree diversity. Ecol. Monogr. 154: 141-164.

Coley, P. 1990. Tasas de Herbivorismo de diferentes Arboles Tropicales. In: E. Leigh, G. Rand \& D. Windsor (eds). p. 191-200. Ecología de un Bosque Tropical. Ciclos Estacionales y Cambios a Largo Plazo. Smithsonian Tropical Research Institute. Balboa, Panamá.

Davis, M., K. Wrage, P. Reiich, M. Tjoelker, T. Schaeffer, \& C. Muermann. 1999.

Survival, growth, and photosynthesis of tree seedlings competing with herbaceous vegetation along a waterlight-nitrogen gradient. Plant Ecol. 145: 341-350.

Dirzo, R. \& J.L. Harper. 1980. Experimental studies on slug-plant interactions: II. The effect of grazing by slugs on high-density monocultures of Capsella bursa-pastoris and Poa апnиа. J. Ecol. 68:999-1011.

Dirzo, R. 1987. Estudios sobre interacciones planta-herbívoro en Los Tuxtlas, Veracruz. Rev. Biol. Trop. 35(Supl.1): 119- 131.

Dirzo, R. \& F.S. Chapin III. 1987. Algunos Problemas Importantes en el Estudio de las

Interacciones Planta-Herbívoro en los Bosques Tropicales. Rev. Biol. Trop. 35(Supl.1): 207-212

Fenner, M. 1983. Relationships between seed weight, ash content and seedling growth in twenty-four species of Compositae. New Phytol. 95: 697-706.

Fenner, M. 1985. Seed ecology. Chapman and Hall, Nueva York, EEUU. 151 p.

García, G. \& R. Dirzo. 2001. Patterns of leaf-pathogen infection in the understory of a Mexican rain forest: incidence, spatiotemporal variation, and mechanisms of infection. Am. J. Bot. 88: 634-645.

García, M. \& O. Morales, 1997. Efecto del extracto acuoso de las hojas de copey, Clusia coclensis (Clusiaceae) sobre la respuesta del reflejo barorreceptor en ratas. Rev. Biol. Trop. 45: 999-1004.

Garwood, N.C. 1990. Ciclo estacional de germinación de semillas en un bosque semicaducifolio tropical. p.243-255. In: E. Leigh, G. Rand \& D. Windsor (eds). Ecología de un bosque tropical. Ciclos estacionales y cambios a largo plazo. Smithsonian Tropical Research Institute. Balboa, Panamá. 
Gilbert, G.S., S.P. Hubell \& R.B. Foster. 1994. Density and distance-to-adult effects of a canker disease of trees in a moist tropical forest. Oecologia 98: 100-108.

Gilbert, G.S. 2002. Evolutionary ecology of plant diseases in natural ecosystems. Annu. Rev. Phytopathol. 40:13-43.

Halter, R., R. Sands \& D.H. Ashton. 1997. Root growth of subalpine and montane Eucalyptus seedlings. Trees 12: $35-41$.

Hammond, D.S. \& V.K. Brown. 1998. Disturbance, phenology and life-history characteristics: factors influencing distance/density-dependent attack on tropical seeds and seedlings. p.51-78. In: D.M. Newbery, H.H.T. Prins, \& N.D. Brown (eds). Dynamics of Tropical Communities, Blackwell, Oxford, Inglaterra.

Holtum, J.A., J.A. Aranda, A. Virgo, H.H. Gehrig \& K. Winter. 2004. $113 \mathrm{C}$ Values and Crassulacean acid metabolism in Clusia species from Panama. Trees 18: $558-668$

Hulme, P.E. 1996. Herbivory, plant regeneration, and species coexistences. J. Ecol. 84: 609-615.

Janzen, D. 1970. Herbivores and the number of tree species in the tropical forest. Am. Nat. 104: 501-528.

Körner, C. \& G. Hoch. 2006. A test of treeline theory on a montane permafrost Island. Arct. Antarct.Alp. Res. 38:113-119.

Kursar, T.A. \& Coley, P.D. 2003. Convergence in defense síndromes of young leaves in tropical rainforest. Biochem. Syst. Ecol. 31: 929-949.

Lajzerowicz, C.C., M. B. Walters, M. Krasowski \& H.B. Massicotte. 2004. Light and temperature differentially colimit subalpine fir and Engelmann spruce seedling growth in partial-cut subalpine forests. Can. J. Forest Res. 34: 249-260.

Leigh, E. 1990. ¿Porqué hay Tantos Tipos de Arboles tropicales? p.175-178. In: E. Leigh,

G. Rand \& D. Windsor (eds). Ecología de un bosque tropical. Ciclos estacionales y cambios a largo plazo. Smithsonian Tropical Research Institute. Balboa, Panamá.

Li, Q. \& K. Ma, 2003. Factors affecting establishment of Quercus iaotungensis Koidz under mature mixed oak forest overstory and in shrubland. Forest. Ecol. Manag. 176: 133-146.

Lüttge, U. 1997. Physiological ecology of tropical plants. Springer-Verlag, Berlín y Heidelberg, Alemania.
Maaron, J. 1997. Interespecific competition and insect herbivory reduce bush lupine (Lupinus arboreus) seedling survival. Oecologia 110: 284-290.

Meiners, S.J. \& S.N. Handel. 2000. Additive and nonadditive effects of herbivory and competition on tree seedling mortality, growth and allocation. Am. J. Bot. 87: 1821-1826.

Meiners, S.J, S.N. Handel, \& S. Pickett. 2000. Tree seedling establishment under insect herbivory: edge effects and interannual variation. Plant Ecol. 151: 161-170.

Mora, L.E. \& H. Sturm, 1995. Estudios Ecológicos de Páramo y del Bosque altoandino en la Cordillera Oriental de Colombia. Academia Colombiana de Ciencias Exactas, Físicas y Naturales. Guadalupe Ltda. Bogotá, Colombia.

Moreno, B. \& J.E. Luque. 1995. Evaluación preliminar de la actividad antialimentaria de seis especies vegetales nativas de Cundinamarca. Entomólogo 39: 1-4.

Nava, V. \& S. Del Amo. 1985. Variación del grado de daño en las hojas de estados juveniles de tres especies primarias, p 299-312. In: A. Gómez-Pompa \& S. Del Amo (eds). Investigaciones sobre la regeneración de selvas altas en Veracruz. Vol II. Instituto Nacional de Investigaciones sobre los Recursos Bióticos. Eds. Alhambra, México D.F.

Núñez-Farfan, J. \& R. Dirzo. 1985. Herbivoría y sucesión de una selva alta perennifolia. p. 313-332. In: A. Gómez-Pompa \& S. Del Amo (eds). Investigaciones sobre la regeneración de selvas altas en Veracruz. Vol II. Instituto Nacional de Investigaciones sobre los Recursos Bióticos. Eds. Alhambra, Distrito Federal, México.

Olivares, E. 1997. Prolonged senescence in Clusia multiflora H.B.K. Trees 11: 370-377.

Salisbury, F. \& C. Ross. 1992. Fisiología Vegetal. Iberoamericana. México.

Silvertown, J. 1987. Introduction to plant population ecology. $2^{\mathrm{a}}$ edición. Longman Scientific \& Technical, Nueva York, EEUU. 229 p.

Sokal, R.R. \& F. Rohlf, 1997. Biometry: the principles and practice of statistics in biological research. W.H. Freeman, Nueva York, EEUU.

Stadler, B., S. Solinger \& M. Beate. 2001. Insect herbivores and the nutrient flow from the canopy to the soil in coniferous and deciduous forest. Oecologia 126: 104-113. 
Sullivan, J.J. 2003. Density-dependent shoot-borer herbivory increases the age of first reproduction and mortality of neotropical tree saplings. Oecologia 136: 96-106.

Swaine, M.D. 1989. Population Dynamics of Tree Species in Tropical Forest. p101-112. In:

L.B. Holm-Nielsen, I.C. Nielsen \& H. Balslev (eds.) Tropical forest, botanical dynamics, speciation and diversity. Academic Press, Londres, United Kingdom.

Tesfaye, G., D. Teketay \& M. Fetene. 2002. Regeneration of fourteen tree species in Harenna forest, southeastern Ethiopia. Flora 197: 461-474.

Tozer, M.G. \& Bradstock, R.A. 1997. Factors influencing the establishment of seedlings of the mallee, Eucaliptus luehmanniana (Myrtaceae). Aust. J. Bot. 45: 997-1008.
Tyree, M., V. Velez \& W. Dalling. 1998. Growth dynamics of root and shoot hydraulic conductance in seedlings of five neotropical tree species: scaling to show possible adaptation to different light regimes. Oecologia 114: 293-298.

Vargas, O. \& S. Zuluaga, 1981. Contribución al estudio fitoecológico de la región de

Monserrate: ecosistemas altoandinos. Tesis. Departamento de Biología, Facultad de Ciencias Universidad Nacional de Colombia.

Vargas, O. 1986. Estudios Ecológicos en un Relicto de Bosque de Weinmannia tomentosa y Drimys granadensis en la región de Monserrate. Pérez Arbelaezia 1: 337-356.

Zar, J.H. 1999. Biostatistical Analysis. 4a edición.. Prentice Hall, Nueva Jersey, EEUU. 